\title{
Reversal of the magnetoelectric effect at a ferromagnetic metal/ ferroelectric interface induced by metal oxidation
}

\author{
Zhaojin Chen ${ }^{1}$, Qiong Yang $\mathbb{1}^{1 凶}{ }^{凶}$, Lingling Tao $\mathbb{1}^{2 凶}$ and Evgeny Y. Tsymbal $\mathbb{1}^{2 凶}$
}

Multiferroic materials composed of ferromagnetic and ferroelectric components are interesting for technological applications due to sizable magnetoelectric coupling allowing the control of magnetic properties by electric fields. Due to being compatible with the silicon-based technology, $\mathrm{HfO}_{2}$-based ferroelectrics could serve as a promising component in the composite multiferroics. Recently, a strong charge-mediated magnetoelectric coupling has been predicted for a $\mathrm{Ni} / \mathrm{HfO}_{2}$ multiferroic heterostructure. Here, using density functional theory calculations, we systematically study the effects of the interfacial oxygen stoichiometry relevant to experiments on the magnetoelectric effect at the $\mathrm{Ni} / \mathrm{HfO}_{2}$ interface. We demonstrate that the magnetoelectric effect is very sensitive to the interface stoichiometry and is reversed if an oxidized Ni monolayer is formed at the interface. The reversal of the magnetoelectric effect is driven by a strong $\mathrm{Ni}-\mathrm{O}$ bonding producing exchange-split polarization-sensitive antibonding states at the Fermi energy. We argue that the predicted reversal of the magnetoelectric effect is typical for other $3 d$ ferromagnetic metals, such as $\mathrm{Co}$ and $\mathrm{Fe}$, where the metal-oxide antibonding states have an opposite spin polarization compared to that in the pristine ferromagnetic metals. Our results provide an important insight into the mechanism of the interfacial magnetoelectric coupling, which is essential for the physics and application of multiferroic heterostructures.

npj Computational Materials (2021)7:204; https://doi.org/10.1038/s41524-021-00679-2

\section{INTRODUCTION}

Multiferroic materials have drawn much attention due to the magnetoelectric coupling providing new paradigms for electronic devices $^{1,2}$. The control of magnetic properties by an applied electric field rather than a magnetic field or a spin-polarized current requires much less energy ${ }^{3-5}$, making multiferroics promising for non-volatile memory and logic applications ${ }^{6,7}$. Layered multiferroic structures comprising ferroelectric and ferromagnetic components are most favorable in this regard owing to the significant interfacial magnetoelectric coupling strength, which can be a few orders of magnitude larger than that in the single-phase multiferroics ${ }^{8-11}$, as a result of the strainmediated $^{12-16}$ and charge-mediated ${ }^{17-23}$ coupling mechanisms. In addition, the magnetoelectric effect in the ferromagnet/ferroelectric heterostructures can be employed in multiferroic tunnel junctions-non-volatile multilevel electronic devices ${ }^{24-29}$.

Over the last years, a wide range of multiferroic materials and structures have been assessed for technological applications ${ }^{2}$. Within these efforts, the challenge was the compatibility of these materials with silicon-based technologies ${ }^{30}$. Most of the traditional perovskite ferroelectrics are incompatible with the complementary metal-oxide-semiconductor (CMOS) technology. In addition, ferroelectric properties of perovskites oxides often deteriorate with reduced film thickness that makes a composite structure impractical at the nanoscale.

Recently, ferroelectricity has been discovered in doped hafnia $\left(\mathrm{HfO}_{2}\right)$ films ${ }^{31,32}$, which may help to address the abovementioned challenges. The origin of ferroelectric behavior was attributed to a non-centrosymmetric orthorhombic phase of $\mathrm{HfO}_{2}$ with the space group symmetry $\mathrm{Pca}_{2}{ }^{33,34}$ Due to robust ferroelectricity at the nanometer scale and good compatibility with the CMOS technology, $\mathrm{HfO}_{2}$-based ferroelectrics have attracted significant attention ${ }^{35-37}$. Considerable efforts have been invested in studying relevant properties to explore their applicability to non-volatile memory devices, such as ferroelectric random-access memories $(\text { FeRAM })^{38}$, ferroelectric field-effect transistors $(\text { FeFET })^{39,40}$, ferroelectric tunnel junctions $(\mathrm{FTJS})^{41-46}$, and negative-capacitance field-effect transistors (NCFET) ${ }^{47,48}$.

The $\mathrm{HfO}_{2}$-based ferroelectric thin films are also promising as a ferroelectric component in multiferroic heterostructures for the high-density memory applications ${ }^{49-52}$. Based on density functional theory (DFT) calculations, a strong polarization-controlled magnetoelectric coupling has been predicted at the interface between ferromagnetic metal $\mathrm{Ni}$ and ferroelectric $\mathrm{HfO}_{2}{ }^{49}$. Stimulated by this prediction, experimental studies of the magnetoelectric coupling at the $\mathrm{Ni} / \mathrm{Hf}_{0.5} \mathrm{Zr}_{0.5} \mathrm{O}_{2}$ ( $\mathrm{HZO}$ ) interface have been conducted and revealed a sizable effect ${ }^{52}$. The ferroelectric polarization control of magnetocrystalline anisotropy and a coercive field have been demonstrated in ultrathin Aldoped- $\mathrm{HfO}_{2} / \mathrm{Co} / \mathrm{Pt}$ heterostructures ${ }^{50}$. A sizable tunneling magnetoresistance (TMR) has been observed in $\mathrm{La}_{0.7} \mathrm{Sr}_{0.3} \mathrm{MnO}_{3}$ (LSMO)/ $\mathrm{Hf}_{0.5} \mathrm{Zr}_{0.5} \mathrm{O}_{2}(\mathrm{HZO}) / \mathrm{Co}$ magnetic tunnel junctions (MTJs) ${ }^{51}$. Interestingly, the TMR effect in these studies was reversed (i.e., the TMR changed from positive to negative) after a few tens of electric field cycling under the same DC bias. In addition, in a separate study, it was found that a $\mathrm{CoO}_{x}$ layer formed at the $\mathrm{Co} / \mathrm{Hf}_{0.5} \mathrm{Zr}_{0.5} \mathrm{O}_{2}$ interface after $\sim 100$ times of electric field cycling, leading to a high-resistance state in LSMO/HZO/Co multiferroic tunnel junctions ${ }^{53}$.

The latter results indicate that the effects of ferromagnetic metal oxidation play an important role in functional multiferroic devices. It is well known that oxygen ions in oxide materials can easily diffuse into the adjacent metal film under applied voltage causing oxidation of metal surface at the metal/oxide interfaces. The formation of an oxide layer produces a significant effect on magnetic properties, such as the interface magnetization, the

${ }^{1}$ School of Materials Science and Engineering, Xiangtan University, Xiangtan, Hunan 411105, China. ${ }^{2}$ Department of Physics and Astronomy, University of Nebraska, Lincoln, NE 68588, USA. ${ }_{\text {email: }}$ qyang@xtu.edu.cn; Iltaophys@gmail.com; tsymbal@unl.edu 
magnetocrystalline anisotropy, the spin-orbit torque, and the TMR effect $^{54-59}$. For example, it has been shown that the oxidation of the Co metal surface results in the formation of the $\mathrm{Co}-\mathrm{O}$ bonding or antibonding states, which modifies the electronic and magnetic structure and leads to the reversal of the TMR effect ${ }^{60,61}$.

Since oxidation of a ferromagnetic metal is expected to occur in realistic devices based on ferromagnet/ferroelectric $\mathrm{HfO}_{2}$ interfaces $^{52}$, in this work, we systematically explore the effects of interfacial oxygen stoichiometry on magnetoelectric properties. We consider a $\mathrm{Ni} / \mathrm{HfO}_{2}$ heterostructure as a prototype model and, using first-principles DFT calculations, study the influence of metal oxidation and oxygen deficiency in $\mathrm{HfO}_{2}$ on the magnetoelectric coupling. Consistent with our previous work ${ }^{49}$, we find that the pristine $\mathrm{Ni} / \mathrm{HfO}_{2}$ interface exhibits a "conventional" magnetoelectric effect driven by the depletion (accumulation) of the screening electronic charges, where the interfacial magnetization is enhanced (reduced) for ferroelectric polarization of $\mathrm{HfO}_{2}$ pointing away from (into) the ferromagnetic Ni layer (Fig. 1a, b). When the oxygen deficiency at the interface increases, the reduced charge transfer between $\mathrm{Ni}$ and $\mathrm{O}$ atoms reduces the magnetoelectric coupling due to a smaller contribution to it from the $\mathrm{Ni}-\mathrm{O}$ hybridization at the interface. Surprisingly, we find that the polarization-induced interfacial magnetoelectric effect is reversed when the interfacial $\mathrm{Ni}$ layer is oxidized by diffused oxygen ions from $\mathrm{HfO}_{2}$ (Fig. 1c, d). We demonstrate that the reversed interface magnetoelectric effect is driven by the formation of the exchange-split $\mathrm{Ni}-\mathrm{O}$ antibonding states, which are sensitive to ferroelectric polarization.

\section{RESULTS}

\section{Interface structures and magnetic properties}

To explore magnetoelectric properties of the $\mathrm{Ni} / \mathrm{HfO}_{2}$ interfaces, we follow our previous work ${ }^{49}$ and consider $\mathrm{Ni} / \mathrm{HfO}_{2} / \mathrm{Ni}$ heterostructures by stacking 5.75 unit cell layers of $\mathrm{HfO}_{2}$ and 9 monolayers of $\mathrm{Ni}$ along the (001) direction. Here, one unit cell layer of $\mathrm{HfO}_{2}$ consists of $2 \mathrm{Hf}$ monolayers and $2 \mathrm{O}$ monolayers. We assume symmetric Ni/O interface terminations, which allows us to examine magnetic properties of the $\mathrm{Ni} / \mathrm{HfO}_{2}(001)$ interface for polarization pointing into the Ni layer or away from the Ni layer, using the same structural model with unidirectional polarization of $\mathrm{HfO}_{2}$ (Fig. 2a). The in-plane lattice constant of the $\mathrm{Ni} / \mathrm{HfO} / \mathrm{Ni}$ heterostructure is fixed to the experimental value of yttriumstabilized zirconia ( $a=5.185 \AA$ ) which is a common substrate to grow $\mathrm{HfO}_{2}$-based ferroelectric thin films ${ }^{62,63}$.

The atomic structure of the defect-free (SDF) $\mathrm{Ni} / \mathrm{HfO}_{2} / \mathrm{Ni}$ heterostructure is optimized with the two interfaces shown in Fig. 2a. The ferroelectric polarization of $\mathrm{HfO}_{2}$ is pointing into $\left(P_{\text {in }}\right)$ and away from $\left(P_{\text {out }}\right)$ the $\mathrm{Ni}$ layer for the top and bottom interfaces, respectively. The average magnetic moments of the interfacial $\mathrm{Ni}$ atoms for the $P_{\text {in }}$ and $P_{\text {out }}$ interfaces are calculated to be $0.97 \mu_{\mathrm{B}}\left(m_{\mathrm{in}}\right)$ and $1.24 \mu_{\mathrm{B}}\left(m_{\text {out }}\right)$, respectively (Table 1$)$. Therefore, the magnetization difference $\Delta m$ at the $\mathrm{Ni} / \mathrm{HfO}_{2}$ interface caused by ferroelectric polarization switching is $0.27 \mu_{\mathrm{B}}$ $\left(0.04 \mu_{\mathrm{B}} / \AA^{2}\right)$.

First, we study the effect of oxygen deficiency on the magnetoelectric coupling at the $\mathrm{Ni} / \mathrm{HfO}_{2}$ interface. Figure $2 \mathrm{~b}$ shows the $\mathrm{Ni} / \mathrm{HfO}_{2}$ atomic structure with one oxygen vacancy at each interface (labeled SV-1). Another structure with one oxygen vacancy (labeled SV-1') at the interface is shown in Supplementary Fig. 1b. We find that the SV-1' interface is $0.42 \mathrm{eV}$ higher in the total energy than the SV-1 interface. The average magnetic moments for the SV-1 and SV-1' structures are $0.71 \mu_{\mathrm{B}}$ and $0.93 \mu_{\mathrm{B}}$ for the $P_{\text {in }}$ and $P_{\text {out }}$ interfaces, respectively, resulting in a $\Delta m$ of $0.22 \mu_{\mathrm{B}}$ (Table 1 ). Figure $2 \mathrm{c}$ shows the $\mathrm{Ni} / \mathrm{HfO}_{2}$ atomic structure with two oxygen vacancies at each interface (labeled SV-2), and another structure with two oxygen vacancies (labeled SV-2') is shown in Supplementary Fig. 1c. The SV-2' interface has the total energy of $0.31 \mathrm{eV}$ higher than the SV-2 interface. As seen from Table 1, the magnetization change driven by polarization reversal (a)

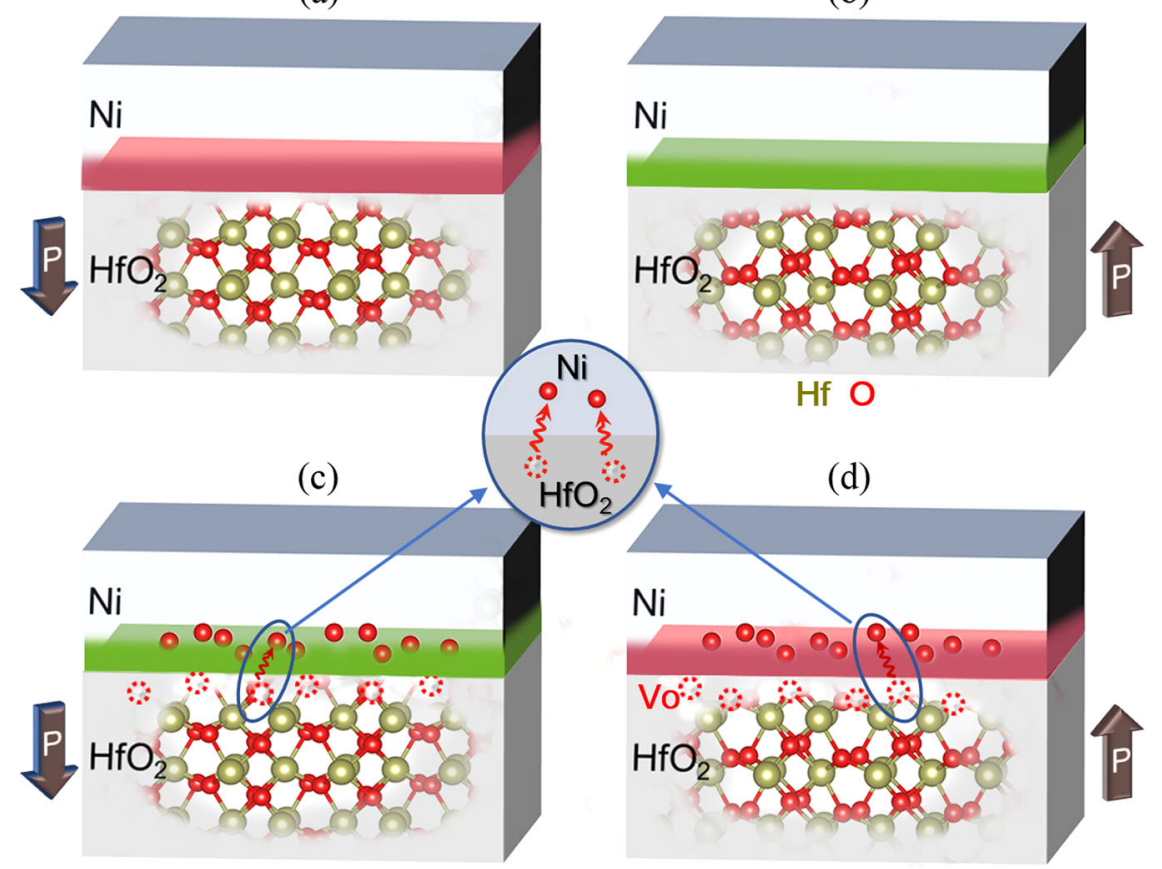

Fig. 1 Magnetoelectric effect reversal induced by interfacial metal oxidation. The ferroelectric-induced interfacial magnetoelectric effect at the pristine defect-free $(\mathbf{a}, \mathbf{b})$ and oxidized-metal $(\mathbf{c}, \mathbf{d}) \mathrm{Ni} / \mathrm{HfO}_{2}$ interface. The vertical arrows represent ferroelectric polarization of the $\mathrm{HfO}{ }_{2}$ layer. Red and green colors at the $\mathrm{Ni} / \mathrm{HfO}_{2}$ interfaces denote an increase or decrease of the interface magnetization induced by the ferroelectric polarization, respectively. Red solid and open spheres at the $\mathrm{Ni} / \mathrm{HfO}_{2}$ interfaces in c and $\mathbf{d}$ indicate the diffused oxygen atoms and the remaining oxygen vacancies (Vo), respectively, which are amplified in the insert. 

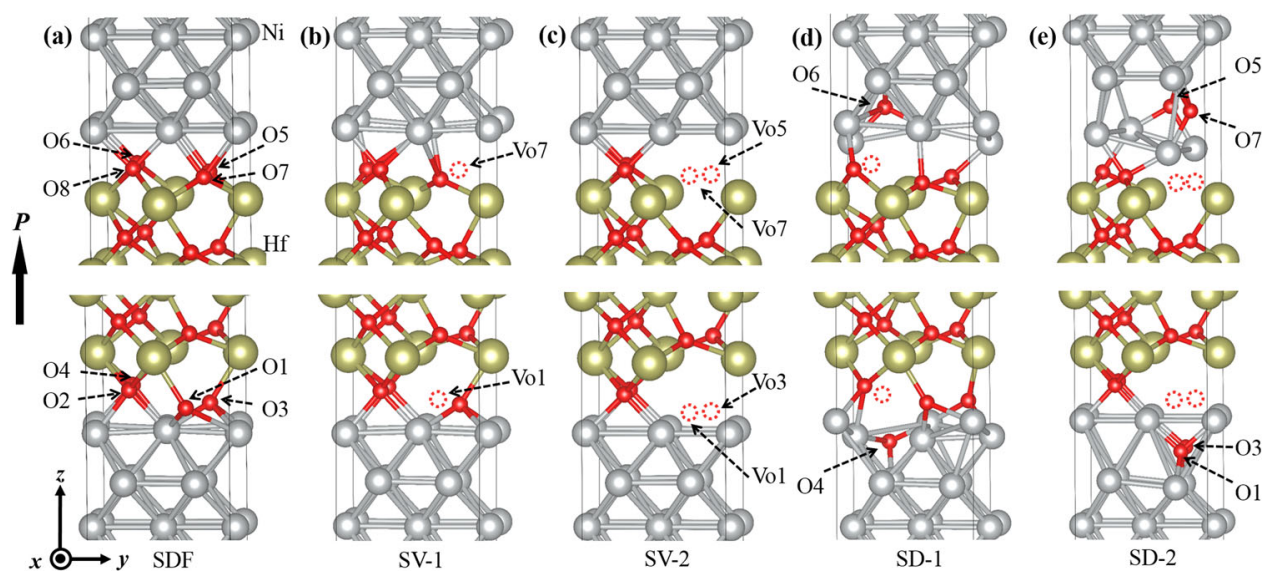

Fig. 2 Atomic structures of the $\mathrm{Ni} / \mathrm{HfO}_{2}$ interfaces with different stoichiometries. The interfaces with polarization pointing away from (into) the $\mathrm{Ni}$ layer are displayed on bottom (top) panels and derived from respective $\mathrm{Ni} / \mathrm{HfO}_{2} / \mathrm{Ni}$ heterostructures with polarization pointing up (indicated by black arrow). a The defect-free $\mathrm{Ni} / \mathrm{HfO}_{2}$ interfaces (SDF). The oxygen atoms at the two interfaces are labeled by $\mathrm{O} 1-\mathrm{O} 8 . \mathrm{O} 1, \mathrm{O} 3$, $\mathrm{O} 5$, and $\mathrm{O} 7$ belong to the $\mathrm{O}$ atoms which are three-fold coordinated in $\mathrm{HfO}_{2}$ bulk and responsible for the ferroelectric displacements. $\mathrm{O} 2$, $\mathrm{O} 4$, $\mathrm{O} 6$, and $\mathrm{O} 8$ belong to the $\mathrm{O}$ atoms which are four-fold coordinated in $\mathrm{HfO}_{2}$ bulk. $\mathbf{b}-\mathbf{e}$ The most stable structures with one oxygen vacancy at each interface (SV-1), with two oxygen vacancies at each interface (SV-2), with one interfacial oxygen atom diffused into Ni (SD-1) and with two interfacial oxygen atoms diffused into $\mathrm{Ni}$ (SD-2), respectively. Red dashed circles indicate the sites of oxygen vacancies.

\begin{tabular}{|c|c|c|c|c|c|c|c|c|c|}
\hline & SDF & SV-1 & SV-1' & SV-2 & SV-2' & SD-1 & SD-1' & SD-2 & SD-2' \\
\hline$m_{\text {out }}$ & 1.24 & 0.93 & 0.93 & 0.66 & 0.62 & 0.90 & 0.70 & 0.03 & 0.17 \\
\hline$m_{\text {in }}$ & 0.97 & 0.71 & 0.71 & 0.54 & 0.44 & 0.58 & 0.59 & 0.38 & 0.29 \\
\hline$\Delta m$ & 0.27 & 0.22 & 0.22 & 0.12 & 0.18 & 0.32 & 0.11 & -0.35 & -0.12 \\
\hline
\end{tabular}

is slightly reduced for the SV-2 $\left(\Delta m=0.12 \mu_{\mathrm{B}}\right)$ and SV-2' $(\Delta m=$ $0.18 \mu_{\mathrm{B}}$ ) structures as compared to that for the SDF, SV-1, and SV-1' structures.

Next, we explore the effect of $\mathrm{Ni}$ surface oxidation on the magnetoelectric coupling by constructing the structures, where the $\mathrm{O}$ atoms are moved inside the interfacial Ni layers forming a $\mathrm{NiO}$ layer. SD-1 (Fig. 2d) and SD-1' (Supplementary Fig. 1d) structures are formed by moving one $\mathrm{O}$ atom inside the interfacial Ni layers. For the SD-1 interface, we find $m_{\text {in }}=0.58 \mu_{\mathrm{B}}$ and $m_{\text {out }}=$ $0.90 \mu_{\mathrm{B}}$, resulting in $\Delta m=0.32 \mu_{\mathrm{B}}$. For the SD-1' interface, which is $0.2 \mathrm{eV}$ higher in energy than the SD-1 interface, $m_{\text {in }}$ and $m_{\text {out }}$ are 0.59 and $0.70 \mu_{\mathrm{B}}$, respectively, resulting in $\Delta m$ of $0.11 \mu_{\mathrm{B}}$ (Table 1). SD-2 (Fig. 2e) and SD-2' (Supplementary Fig. 1e) structures are obtained by moving two $\mathrm{O}$ atoms inside the interfacial Ni layers. We find that the SD-2 interface is $0.17 \mathrm{eV}$ lower in energy than the SD-2' interface. From Table 1, it is seen that the interfacial magnetic moments for the SD-2 and SD- $2^{\prime}$ structures are significantly reduced with $m_{\text {out }}$ for the SD-2 interface being diminished nearly to zero. The calculated $\Delta m$ for the SD-2 or SD-2 structures is negative, indicating the reversal of the magnetoelectric effect with oxidation of $\mathrm{Ni}$.

\section{Interface electronic properties}

The difference in the magnetoelectric effect depending on the interfacial oxygen stoichiometry can be understood from the analysis of the electronic structure. Figure 3 shows the spinresolved density of states (DOS) projected onto the interfacial $\mathrm{Ni}$ atoms and diffused $\mathrm{O}$ for the $\mathrm{Ni} / \mathrm{HfO}_{2} / \mathrm{Ni}$ heterostructures shown in Fig. 2. For the pristine defect-free structure (Fig. 3a), ferroelectric polarization of $\mathrm{HfO}_{2}$ shifts the DOS of the interfacial $\mathrm{Ni}$ atoms at the $P_{\text {out }}\left(P_{\text {in }}\right)$ interface to high (low) energies (compare red and blue lines in Fig. 3a). Since around the Fermi energy the minorityspin DOS of $\mathrm{Ni}$ is much larger than the majority-spin DOS (compare top and bottom panels in Fig. 3a), this shift of the $\mathrm{Ni}$ DOS results in the depletion (accumulation) of the minority-spin electrons and hence an enhanced (reduced) magnetic moment of the $\mathrm{Ni}$ atoms at the $P_{\text {out }}\left(P_{\text {in }}\right)$ interface.

In addition to the electrostatic doping, there is another contribution to the magnetoelectric effect resulting from the electronic hybridization between the interfacial $\mathrm{Ni}$ and $\mathrm{O}$ atoms dependent on polarization orientation. As was explained in our previous work ${ }^{49}$, the weaker (stronger) hybridization between the O $2 p$ and $\mathrm{Ni} 3 d$ orbitals at the $P_{\text {in }}\left(P_{\text {out }}\right)$ interface, due to the longer (shorter) $\mathrm{Ni}-\mathrm{O}$ bonds induced by polar displacements of oxygen atoms at the interface, reduces (enhances) the electron charge transfer from $\mathrm{Ni}$ to $\mathrm{O}$ atoms resulting in the reduced (enhanced) magnetic moments of the interfacial Ni atoms (Supplementary Fig. 2). This effect is largely responsible for the change in the magnetoelectric coupling at the interfaces with one (SV-1 and SV $-1^{\prime}$ ) or two oxygen vacancies (SV-2 and SV-2') compared to the defect-free (SDF) interface. As seen from Table 1 and Supplementary Table 1, removing one or two $\mathrm{O}$ atoms from the interface, gradually decreases the magnetic moments of the $\mathrm{Ni}$ atoms at both $P_{\text {in }}$ and $P_{\text {out }}$ interfaces. This decrease is due to the reduced electron charge transfer from the interfacial $\mathrm{Ni}$ to $\mathrm{O}$ atoms (not related to ferroelectricity) reflected in the diminished overall rightward energy shift of the interfacial Ni DOS relative to the bulk Ni DOS (compare Fig. 3a and b, c which elevates the minority-spin population. The reduced charge transfer between $\mathrm{Ni}$ and $\mathrm{O}$ atoms with the decreased number of interfacial $\mathrm{O}$ atoms reduces the magnitude of the magnetoelectric coupling due to a smaller contribution to it from the $\mathrm{Ni}-\mathrm{O}$ hybridization at the interface.

Diffusion of $\mathrm{O}$ atoms into the Ni layer affects differently the magnetoelectric coupling depending on the degree of the Ni layer oxidation. For the SD-1 and SD-1' structures, where only one $\mathrm{O}$ atom per unit cell is diffused underneath the top Ni monolayer (Fig. $2 \mathrm{~d}$ and Supplementary Fig. 1d), the interfacial Ni DOS (Fig. 3d and Supplementary Fig. 3d) are like those for the structures with one oxygen vacancy at the interface (Fig. $3 \mathrm{~b}$ and Supplementary Fig. 3b), resulting in qualitatively similar magnetoelectric effects. 
4

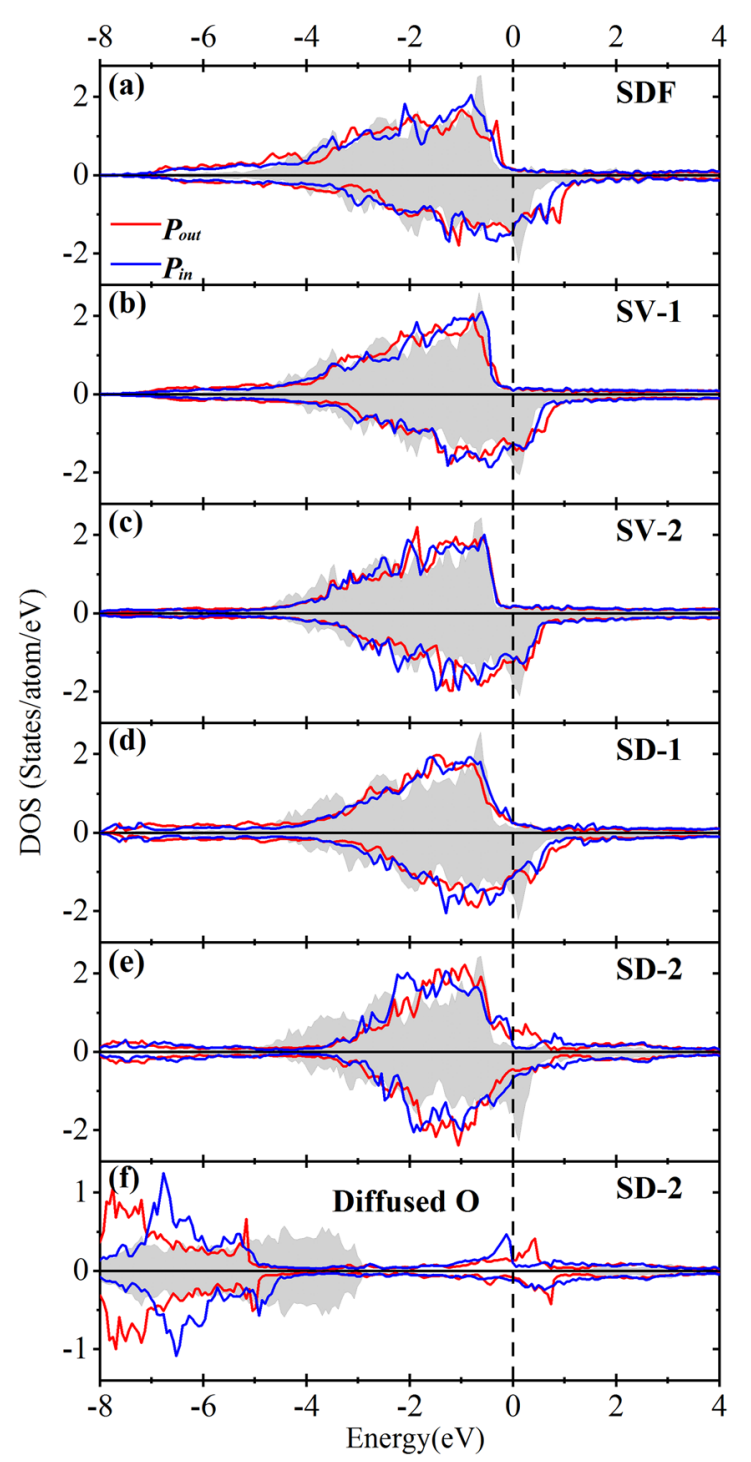

Fig. 3 Spin-resolved density of states of interfacial $\mathrm{Ni}$ and diffused $\mathbf{0}$. a-e Spin-resolved density of states (DOS) of the interfacial $\mathrm{Ni}$ atoms at the $\mathrm{Ni} / \mathrm{HfO}_{2}$ interfaces for polarization pointing into the Ni layer $\left(P_{\text {in }}\right)$ or away from it $\left(P_{\text {out }}\right)$ for a SDF, b SV-1, c SV-2, d SD-1, and e SD-2 structures. $f$ DOS of diffused $O$ atoms at the SD-2 interfaces for $P_{\text {in }}$ and $P_{\text {out }}$. DOS of $\mathrm{Ni}$ and $\mathrm{O}$ atoms under the considered energy range are mainly contributed by the $\mathrm{Ni}$ $3 d$ and $O 2 p$ orbitals, respectively. Each curve represents an averaged DOS per atom. The majority- and minority-spin DOS are plotted on top (positive) and bottom (negative) panels, respectively. The red and blue lines denote DOS at the $P_{\text {out }}$ and $P_{\text {in }}$ interfaces, respectively. The gray-filled curves correspond to the bulk-like DOS of $\mathrm{Ni}$ atoms at the central $\mathrm{Ni}$ layer $\mathbf{a}-\mathbf{e}$ or $\mathrm{O}$ atoms at the central $\mathrm{HfO}_{2}$ layer (f). The vertical dashed line indicates the position of Fermi energy $\left(E_{\mathrm{F}}\right)$.

However, the interfacial Ni DOS changes dramatically for the SD-2 and SD-2' structures where two $\mathrm{O}$ atoms are diffused into the $\mathrm{Ni}$ layer resulting in the formation of the $\mathrm{NiO}$ interfacial monolayer. As seen from Fig. 3e and Supplementary Fig. 3e, the minority-spin DOS around the Fermi energy is significantly reduced for the interfacial $\mathrm{Ni}$ monolayer as compared to the bulk $\mathrm{Ni}$ (gray background in Fig. $3 \mathrm{e}$ and Supplementary Fig. 3e) and the pristine interface (Fig. 3a). This effect stems from the broken bonds between the interfacial and bulk $\mathrm{Ni}$ atoms and the formation of the $\mathrm{Ni}-\mathrm{O}$ antibonding states near the Fermi energy. The antibonding states are clearly seen from the DOS projected onto the diffused $\mathrm{O}$ atoms shown in Fig. 3f. While the bonding states are located at energies around and below $E=-5 \mathrm{eV}$, the exchange-split antibonding states appear in the energy window $-1 \mathrm{eV}<E<+1 \mathrm{eV}$ around the Fermi energy. The reduced minorityspin DOS is responsible for the sizable reduction of the magnetic moment of the $\mathrm{Ni}$ atoms at the interface compared to the pristine SDF structure (Table 1).

The effect of $\mathrm{Ni}$ oxidation resulting in the formation of the $\mathrm{Ni}-\mathrm{O}$ antibonding states leads to the reversal of the magnetoelectric effect. This is due to the exchange-split $\mathrm{Ni}-\mathrm{O}$ antibonding states which cross the Fermi energy with reversal of the ferroelectric polarization of $\mathrm{HfO}_{2}$. The effect is clearly seen from Fig. $3 f$ displaying the portion of the antibonding states near the Fermi energy projected onto the diffused $\mathrm{O}$ atoms. While the minorityspin DOS corresponding to the $\mathrm{Ni}-\mathrm{O}$ antibonding states is largely located above the Fermi energy independent of ferroelectric polarization orientation, the antibonding peak in the majority-spin DOS crosses the Fermi energy when the polarization is reversed. The same effect can be seen from the DOS projected onto the interfacial $\mathrm{Ni}$ atoms (Fig. 3e), though less pronounced due to the overlap in DOS between the antibonding and non-bonding $\mathrm{Ni}$ states. As a result, when the ferroelectric polarization of $\mathrm{HfO}_{2}$ is pointing into the Ni layer $\left(P_{\text {in }}\right)$, the accumulation of the majorityspin electrons (blue line) in the $\mathrm{Ni}-\mathrm{O}$ antibonding states enhances the magnetic moments of the interfacial $\mathrm{Ni}$ and $\mathrm{O}$ atoms. Whereas, when the polarization of $\mathrm{HfO}_{2}$ layer is pointing away from the $\mathrm{Ni}$ layer $\left(P_{\text {out }}\right)$, the depletion of the majority-spin antibonding states (red line) reduces the magnetic moments of the interfacial $\mathrm{Ni}$ and $\mathrm{O}$ atoms. Therefore, the magnetoelectric effect of the oxidized $\mathrm{Ni} /$ $\mathrm{HfO}_{2}$ interface is reversed compared to the pristine defect-free $\mathrm{Ni}$ / $\mathrm{HfO}_{2}$ interface.

The effects of the interface stoichiometry on the interface magnetoelectric effect are visualized using spin-density plots shown in Fig. 4 (see also Supplementary Fig. 4). For the SDF structure (Fig. 4a), the depletion (accumulation) of the minorityspin electrons due to the polarization screening charge and the stronger (weaker) hybridization between the interfacial $\mathrm{O}$ and $\mathrm{Ni}$ atoms induced by $\mathrm{O}$ polar displacements at the $P_{\text {out }}\left(P_{\text {in }}\right)$ interface leads to a larger spin density on the interfacial $\mathrm{Ni}$ and $\mathrm{O}$ atoms at the $P_{\text {out }}$ interface than at the $P_{\text {in }}$ interface. For the SV-2 structure (Fig. $4 \mathrm{~b}$ ), the electron charge transfer from the interfacial $\mathrm{Ni}$ to $\mathrm{O}$ atoms is reduced due to the reduced number of $\mathrm{O}$ atoms at the interface, raising the minority-spin population and thus lowering the spin density as compared to the SDF structure. At the same time, the decreased number of the interfacial $O$ atoms reduces the contribution to the magnetoelectric coupling from the $\mathrm{Ni}-\mathrm{O}$ hybridization at the interface, although the spin density and thus the magnetization at the $P_{\text {out }}$ interface still remain larger than those at the $P_{\text {in }}$ interface. For the SD-2 structure (Fig. 4c), the spin density at both interfaces decreases significantly due to the formation of the NiO layer at the interface. It is seen that the spin density and thus the magnetization are larger at the $P_{\text {in }}$ interface than at the $P_{\text {out }}$ interface, implying that the magnetoelectric effect is reversed at the SD-2 interface compared to the SDF and SV-2 interfaces. The spin densities of the interfaces with other interface stoichiometries demonstrate similar trends (see Supplementary Fig. 4).

\section{DISCUSSION}

We would like to note that the predicted effects of the interface stoichiometry on the magnetoelectric effect at the interfaces between a ferromagnetic metal and an oxide ferroelectric are expected to be not only limited to the considered $\mathrm{Ni} / \mathrm{HfO}_{2}$ interface but may have more general occurrence. In particular, the conventional magnetoelectric effect, where depletion (accumulation) of the screening electronic charges induced by ferroelectric polarization pointing away from (into) the ferromagnetic metal 


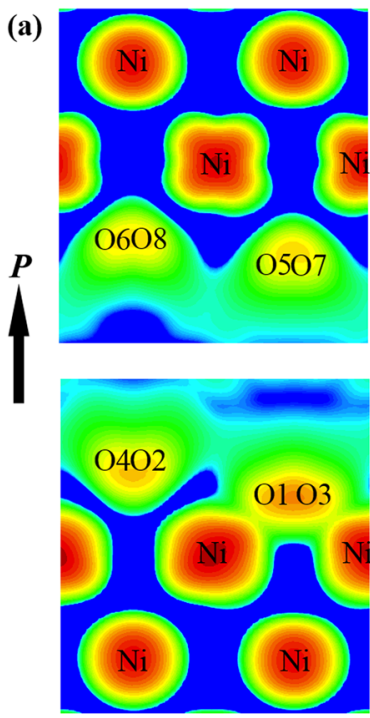

SDF (b)
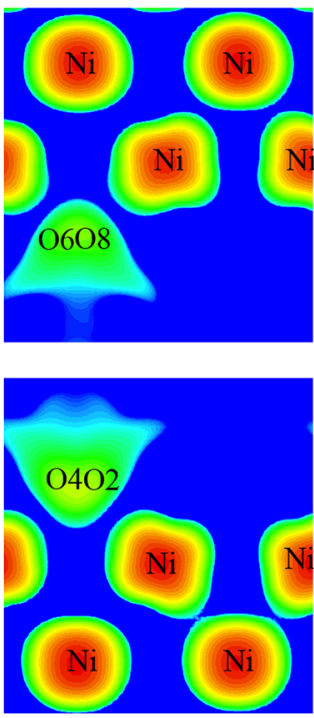

SV-2 (c)
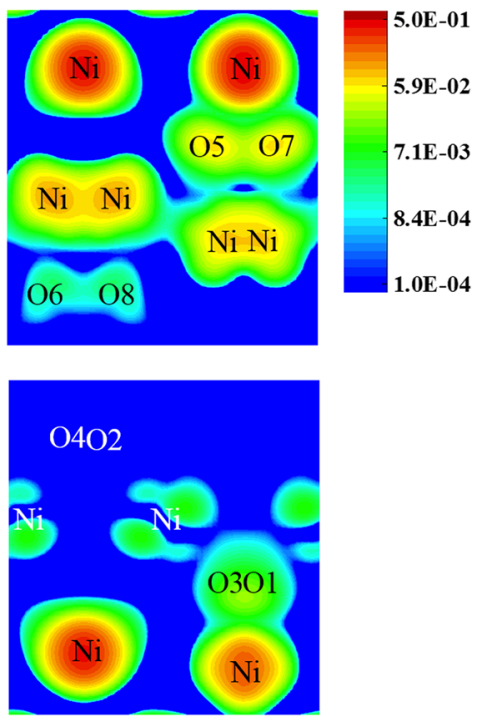

SD-2

Fig. 4 Spin densities. The spin densities of the $\mathrm{Ni} / \mathrm{HfO}_{2}$ interfaces defined as the difference between the majority- and minority-spin charge densities, at the $P_{\text {in }}$ (top panels) and $P_{\text {out }}$ (bottom panels) interfaces for a SDF, b SV-2, and $\mathbf{c}$ SD-2 interfaces. The spin densities are shown within the $y z$-plane and averaged along the $x$-direction.

(a) Conventional magnetoelectric effect

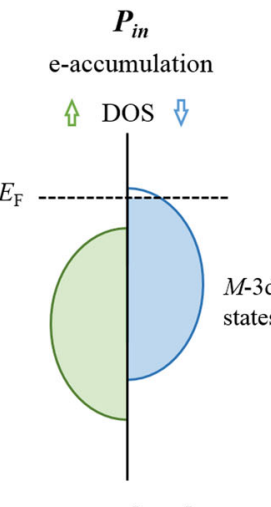

$m$ reduced

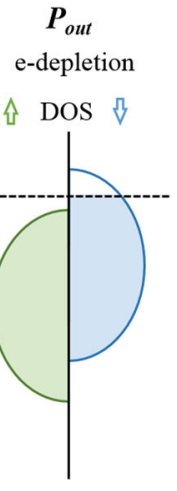

$m$ enhanced (b) Reversed magnetoelectric effect

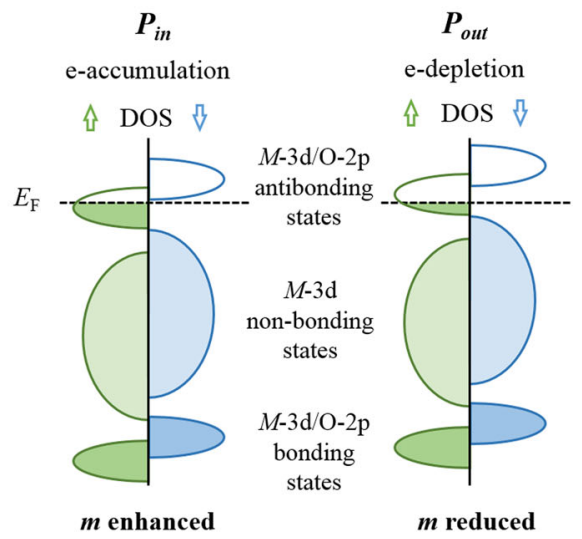

Fig. 5 Schematic of the magnetoelectric effect. a Conventional magnetoelectric effect, where accumulation (depletion) of the screening minority-spin electronic charges induced by ferroelectric polarization pointing into, $\boldsymbol{P}_{\text {in, }}$ (away from, $\boldsymbol{P}_{\text {out }}$ ) the ferromagnetic metal $(M)$ layer leads to reduction (enhancement) of the interfacial magnetic moment $(\boldsymbol{m})$; $\mathbf{b}$ Reversed magnetoelectric effect due to the interface metal oxidation forming $M-3 \mathrm{~d} / \mathrm{O}-2 \mathrm{p}$ bonding and antibonding states, with the latter being positioned at the Fermi energy and controlling the polarization charge screening through accumulation or depletion of the antibonding majority-spin electrons. DOS stays for the density of states and $E_{\mathrm{F}}$ for the Fermi energy. Arrows denote majority (spin-up) and minority (spin-down) DOS.

layer leads to enhancement (reduction) of the interfacial magnetization, is expected to be typical for all elemental $3 \mathrm{~d}$ metals, such as $\mathrm{Fe}, \mathrm{Co}$, and $\mathrm{Ni}$, where the majority $3 \mathrm{~d}$ bands are fully populated resulting in higher minority-spin DOS at the Fermi energy (Fig. 5a). On the other hand, oxidation of these metals at the interface in a metal/ferroelectric heterostructure due to the diffusion of oxygen atoms from the oxide ferroelectric into the ferromagnetic metal forms metal-oxygen bonding and antibonding states with the latter being positioned at the Fermi energy. This may lead to the reversal of the magnetoelectric effect at the interfaces between $3 d$ ferromagnetic metals and ferroelectric oxides due to majority-spin antibonding states controlling the polarization charge screening (Fig. 5b). For example, it has been shown that at the Co surface, the exchange-split Co-O antibonding states reverse the spin polarization compared to that in bulk $\mathrm{Co}^{60,61}$. The effects discussed here may be responsible for the reversal of TMR in LSMO/HZO/Co MTJs after electric field cycling ${ }^{51}$.

From the experimental perspective, the determination of the interface stoichiometry is not a simple problem, especially for the interfaces which are not epitaxial (see, e.g., ref. ${ }^{52}$ ). In this regard, a comparison with the results of DFT calculations, such as those presented here, may be challenging. A valuable approach (though not direct) is to measure a band alignment between a ferromagnetic metal and a ferroelectric oxide using, e.g., X-ray photoelectron spectroscopy ${ }^{64}$ and compare the results with the DFT calculations. Since the valence band offset is very sensitive to the interface termination it can provide valuable information about the interface structure. Valence band offsets for different interface structures are calculated in Supplementary Fig. 5 and Table 2, which may be useful for the experimentalists working in this field. 
In conclusion, the magnetoelectric effects at the $\mathrm{Ni} / \mathrm{HfO}_{2}$ interfaces where ferroelectric polarization of $\mathrm{HfO}_{2}$ controls the interfacial magnetization of ferromagnetic Ni have been studied based on the DFT calculations for different interface stoichiometries. Consistent with the previous studies, it was found that for the ideal fully oxygen terminated $\mathrm{Ni} / \mathrm{HfO}_{2}$ interface, the interfacial magnetization is increased (decreased) when the ferroelectric polarization of $\mathrm{HfO}_{2}$ points away from (into) the $\mathrm{Ni}$ layer by resulting in depletion (accumulation) of the minority-spin electrons of Ni. This conventional interfacial magnetoelectric effect is maintained with reducing the oxygen concentration at the $\mathrm{Ni} / \mathrm{HfO}_{2}$ interface, although its magnitude is diminished. However, when a $\mathrm{NiO}$ monolayer is formed at the $\mathrm{Ni} / \mathrm{HfO}_{2}$ interface due to diffusion of oxygen atoms from the $\mathrm{HfO}_{2}$ surface into the Ni layer, the ferroelectric-induced magnetoelectric effect is reversed. This reversal is driven by the formation of the exchange-split $\mathrm{Ni}-\mathrm{O}$ antibonding states resulting from the $\mathrm{Ni}$ oxidation. Due to the majority-spin $\mathrm{Ni}-\mathrm{O}$ antibonding states controlling the ferroelectric polarization charge screening, the electron accumulation (depletion) enhances (reduces) the interface magnetization, leading to the reversed magnetoelectric effect as compared with that at the unoxidized $\mathrm{Ni} / \mathrm{HfO}_{2}$ interface. The predicted effect of the ferromagnetic metal oxidation on the interfacial magnetoelectric coupling is of general significance, and therefore we expect similar behaviors occurring at other ferromagnetic metal/ferroelectric oxide interfaces. We, therefore, hope that our theoretical predictions will stimulate further experimental studies of the ferroelectrically-induced interfacial magnetoelectric effect and will improve the understanding of this phenomenon.

\section{METHODS \\ DFT calculations}

DFT calculations are performed using the Vienna Ab Initio Simulation Package (VASP) using the projector-enhanced wave (PAW) method ${ }^{65}$. The generalized gradient approximation (GGA) within the Perdew-BurkeErnzerhof (PBE) functional ${ }^{66}$ is used to describe the exchange and correlation effects. A $450 \mathrm{eV}$ plane-wave cutoff and a $6 \times 6 \times 1$ Monkhorst-Pack $k$-point mesh ${ }^{67}$ are used for structural relaxations of the $\mathrm{Ni} / \mathrm{HfO}_{2} / \mathrm{Ni}$ heterostructures and a $10 \times 10 \times 1$ Monkhorst-Pack $k$-point mesh is then used in the self-consistent electronic structure calculation.

\section{DATA AVAILABILITY}

The data that support the findings of the work is in the manuscript's main text and Supplementary Information. Additional data are available from the corresponding author upon reasonable request.

Received: 28 September 2021; Accepted: 28 November 2021; Published online: 14 December 2021

\section{REFERENCES}

1. Eerenstein, W., Mathur, N. \& Scott, J. F. Multiferroic and magnetoelectric materials. Nature 442, 759-765 (2006).

2. Spaldin, N. A. \& Ramesh, R. Advances in magnetoelectric multiferroics. Nat. Mater. 18, 203-212 (2019).

3. Tsymbal, E. Y. Spintronics Electric toggling of magnets. Nat. Mater. 11, 12-13 (2012).

4. Manipatruni, S., Nikonov, D. E. \& Young, I. A. Beyond CMOS computing with spin and polarization. Nat. Phys. 14, 338-343 (2018).

5. Meisenheimer, P. B., Novakov, S., Vu, N. M. \& Heron, J. T. Perspective: magnetoelectric switching in thin film multiferroic heterostructures. J. Appl. Phys. 123, 240901 (2018).

6. Scott, J. F. Data storage: multiferroic memories. Nat. Mater. 6, 256-257 (2007).

7. Bibes, M. \& Barthélémy, A. Multiferroics: towards a magnetoelectric memory. Nat. Mater. 7, 425-426 (2008).

8. Martin, L. W. et al. Multiferroics and magnetoelectrics: thin films and nanostructures. J. Phys.: Condens. Matter 20, 434220 (2008).

9. Velev, J. P., Jaswal, S. S. \& Tsymbal, E. Y. Multi-ferroic and magnetoelectric materials and interfaces. Philos. Trans. R. Soc. A 369, 3069 (2011).
10. Vaz, C. A. F. Electric field control of magnetism in multiferroic heterostructures. J. Phys.: Condens. Matter 24, 333201 (2012).

11. Hu, J.-M., Duan, C.-G., Nan, C.-W. \& Chen, L.-Q. Understanding and designing magnetoelectric heterostructures guided by computation: progresses, remaining questions, and perspectives. npj Comp. Mater. 3, 18 (2017).

12. Nan, C.-W., Bichurin, M. I., Dong, S., Viehland, D. \& Srinivasan, G. Multiferroic magnetoelectric composites: historical perspective, status, and future directions. J. Appl. Phys. 103, 031101 (2008).

13. Nan, T. X. et al. Voltage impulse induced bistable magnetization switching in multiferroic heterostructures. Appl. Phys. Lett. 100, 132409 (2012).

14. Franke, K. J. A., López González, D., Hämäläinen, S. J. \& van Dijken, S. Size dependence of domain pattern transfer in multiferroic heterostructures. Phys. Rev. Lett. 112, 017201 (2014).

15. Wang, Q. et al. Strain-mediated $180^{\circ}$ switching in CoFeB and Terfenol-D nanodots with perpendicular magnetic anisotropy. Appl. Phys. Lett. 110, 102903 (2017).

16. Motti, F. et al. Strain-induced magnetization control in an oxide multiferroic heterostructure. Phys. Rev. B 97, 094423 (2018).

17. Duan, C. G., Jaswal, S. S. \& Tsymbal, E. Y. Predicted magnetoelectric effect in $\mathrm{Fe} / \mathrm{BaTiO}_{3}$ multilayers: ferroelectric control of magnetism. Phys. Rev. Lett. 97, 047201 (2006).

18. Yamauchi, K., Sanyal, B. \& Picozzi, S. Interface effects at a half-metal/ferroelectric junction. Appl. Phys. Lett. 91, 062506 (2007).

19. Molegraaf, H. J. A. et al. Magnetoelectric effects in complex oxides with competing ground states. Adv. Mater. 21, 3470 (2009).

20. Radaelli, G. et al. Electric control of magnetism at the $\mathrm{Fe} / \mathrm{BaTiO}$ interface. Nat. Commun. 5, 3404 (2014).

21. Zhou, Z. et al. Interfacial charge-mediated non-volatile magnetoelectric coupling in $\mathrm{Co}_{0.3} \mathrm{Fe}_{0.7} / \mathrm{Ba}_{0.6} \mathrm{Sr}_{0.4} \mathrm{TiO}_{3} / \mathrm{Nb}: \mathrm{SrTiO}_{3}$ multiferroic heterostructures. Sci. Rep. 5, 7740 (2015).

22. Rajapitamahuni, A. et al. Ferroelectric polarization control of magnetic anisotropy in $\mathrm{PbZr}_{0.2} \mathrm{Ti}_{0.8} \mathrm{O}_{3} / \mathrm{La}_{0.8} \mathrm{Sr}_{0.2} \mathrm{MnO}_{3}$ heterostructures. Phys. Rev. Mater. 3, 021401 (2019).

23. Bocirnea, A. E. et al. Polarization-dependent magnetism of the $\mathrm{Ni} / \mathrm{BaTiO}_{3}$ interface. Phys. Rev. Mater. 4, 034402 (2020).

24. Velev, J. P. et al. Magnetic tunnel junctions with ferroelectric barriers: prediction of four resistance states from first-principles. Nano Lett. 9, 427 (2009).

25. Zhuravlev, M. Y., Maekawa, S. \& Tsymbal, E. Y. Effect of spin-dependent screening on tunneling electroresistance and tunneling magnetoresistance in multiferroic tunnel junctions. Phys. Rev. B 81, 104419 (2010).

26. Pantel, D., Goetze, S., Hesse, D. \& Alexe, M. Reversible electrical switching of spin polarization in multiferroic tunnel junctions. Nat. Mater. 11, 289-293 (2012).

27. Velev, J. P., Burton, J. D., Zhuravlev, M. Y. \& Tsymbal, E. Y. Predictive modelling of ferroelectric tunnel junctions. npj Comp. Mater. 2, 16009 (2016).

28. Huang, W. et al. Solid-state synapse based on magnetoelectrically coupled memristor. ACS Appl. Mater. Interfaces 10, 5649-5656 (2018).

29. Fang, M. et al. Nonvolatile multilevel states in multiferroic tunnel junctions. Phys. Rev. Appl. 12, 044049 (2019).

30. Vopson, M. M. Fundamentals of multiferroic materials and their possible applications. Crit. Rev. Solid State 40, 223-250 (2015).

31. Böscke, T. S., Müller, J., Bräuhaus, D., Schröder, U. \& Böttger, U. Ferroelectricity in hafnium oxide thin films. Appl. Phys. Lett. 99, 102903 (2011).

32. Müller, J. et al. Ferroelectricity in simple binary $\mathrm{ZrO}_{2}$ and $\mathrm{HfO}_{2}$. Nano Lett. 12, 4318-4323 (2012).

33. Huan, T. D., Sharma, V., Rossetti, G. A. Jr. \& Ramprasad, R. Pathways towards ferroelectricity in hafnia. Phys. Rev. B 90, 064111 (2014).

34. Sang, X., Grimley, E. D., Schenk, T., Schroeder, U. \& LeBeau, J. M. On the structural origins of ferroelectricity in $\mathrm{HfO}_{2}$ thin films. Appl. Phys. Lett. 106, 162905 (2015).

35. Jiang, P. et al. Wake-up effect in $\mathrm{HfO}_{2}$-based ferroelectric films. Adv. Electron. Mater. 7, 2000728 (2020).

36. Khan, A. I., Keshavarzi, A. \& Datta, S. The future of ferroelectric field-effect transistor technology. Nat. Electron. 3, 588-597 (2020).

37. Chouprik, A., Negrov, D., Tsymbal, E. Y. \& Zenkevich, A. Defects in ferroelectric $\mathrm{HfO}_{2}$. Nanoscale 13, 11635 (2021).

38. Francois, $\mathrm{T}$. et al. Demonstration of BEOL-compatible ferroelectric $\mathrm{Hf}_{0.5} \mathrm{Zr}_{0.5} \mathrm{O}_{2}$ scaled FeRAM co-integrated with $130 \mathrm{~nm}$ CMOS for embedded NVM applications. IEEE IEDM (2019). https://doi.org/10.1109/IEDM19573.2019.8993485.

39. Mueller, S. et al. From MFM capacitors toward ferroelectric transistors: endurance and disturb characteristics of $\mathrm{HfO}_{2}$-based FeFET devices. IEEE T. Electron Dev. 60, 4199-4205 (2013).

40. Seo, M. et al. First demonstration of a logic-process compatible junctionless ferroelectric FinFET synapse for neuromorphic applications. IEEE Electr. Device Lett. 39, 1445-1448 (2018)

41. Ambriz-Vargas, F. et al. A complementary metal oxide semiconductor processcompatible ferroelectric tunnel junction. ACS Appl. Mater. Interfaces 9, 13262-13268 (2017).

42. Chouprik, A. et al. Electron transport across ultrathin ferroelectric $\mathrm{Hf}_{0.5} \mathrm{Zr}_{0.5} \mathrm{O}_{2}$ films on Si. Microelectron. Eng. 178, 250-253 (2017). 
43. Goh, Y. \& Jeon, S. Enhanced tunneling electroresistance effects in HfZrO-based ferroelectric tunnel junctions by high-pressure nitrogen annealing. Appl. Phys. Lett. 113, 052905 (2018).

44. Yoong, $\mathrm{H}$. Y. et al. Epitaxial ferroelectric $\mathrm{Hf}_{0.5} \mathrm{Zr}_{0.5} \mathrm{O}_{2}$ thin films and their implementations in memristors for brain-inspired computing. Adv. Funct. Mater. 28, 1806037 (2018).

45. Chen, L. et al. Ultra-low power $\mathrm{Hf}_{0.5} \mathrm{Zr}_{0.5} \mathrm{O}_{2}$ based ferroelectric tunnel junction synapses for hardware neural network applications. Nanoscale 10, 15826-15833 (2018).

46. Mikheev, V. et al. Ferroelectric second-order memristor. ACS Appl. Mater. Interfaces 11, 32108-32114 (2019).

47. Cao, W. \& Banerjee, K. Is negative capacitance FET a steep-slope logic switch? Nat. Commun. 11, 1196 (2020).

48. Hoffmann, M. et al. Unveiling the double-well energy landscape in a ferroelectric layer. Nature 565, 464-467 (2019).

49. Yang, Q. et al. Magnetoelectric effect at the $\mathrm{Ni} / \mathrm{HfO}_{2}$ interface induced by ferroelectric polarization. Phys. Rev. Appl. 12, 024044 (2019).

50. Vermeulen, B. F. et al. Ferroelectric control of magnetism in ultrathin $\mathrm{HfO}_{2} / \mathrm{Co} / \mathrm{Pt}$ layers. ACS Appl. Mater. Interfaces 11, 34385-34393 (2019).

51. Wei, Y. et al. Magneto-ionic control of spin polarization in multiferroic tunnel junctions. npj Quantum Mater. 4, 62 (2019).

52. Dmitriyeva, A. et al. Magnetoelectric coupling at the $\mathrm{Ni} / \mathrm{Hf}_{0.5} \mathrm{Zr}_{0.5} \mathrm{O}_{2}$ interface. ACS Nano 15, 14891-14902 (2021).

53. Nukala, P. et al. Reversible oxygen migration and phase transitions in hafniabased ferroelectric devices. Science 372, 630-635 (2021).

54. Rodmacq, B., Manchon, A., Ducruet, C., Auffret, S. \& Dieny, B. Influence of thermal annealing on the perpendicular magnetic anisotropy of $\mathrm{Pt} / \mathrm{Co} / \mathrm{AlO}_{x}$ trilayers. Phys. Rev. B 79, 024423 (2009).

55. $\mathrm{Bi}, \mathrm{C}$. et al. Reversible control of Co magnetism by voltage-induced oxidation. Phys. Rev. Lett. 113, 267202 (2014).

56. Bauer, U. et al. Magneto-ionic control of interfacial magnetism. Nat. Mater. 14, 174-181 (2015).

57. Qiu, X. et al. Spin-orbit-torque engineering via oxygen manipulation. Nat. Nanotechnol. 10, 333-338 (2015).

58. Hibino, Y., Hirai, T., Hasegawa, K., Koyama, T. \& Chiba, D. Enhancement of the spin-orbit torque in a Pt/Co system with a naturally oxidized Co layer. Appl. Phys. Lett. 111, 132404 (2017).

59. Feng, J. et al. Effects of oxidation of top and bottom interfaces on the electric, magnetic, and spin-orbit torque properties of $\mathrm{Pt} / \mathrm{Co} / \mathrm{AlO}_{x}$ trilayers. Phys. Rev. Appl. 13, 044029 (2020).

60. Belashchenko, K. D. et al. Effect of interface bonding on spin-dependent tunneling from the oxidized Co surface. Phys. Rev. B 69, 174408 (2004).

61. Belashchenko, K. D., Tsymbal, E. Y., Oleynik, I. I. \& van Schilfgaarde, M. Positive spin polarization in $\mathrm{Co} / \mathrm{Al}_{2} \mathrm{O}_{3} / \mathrm{Co}$ tunnel junctions driven by oxygen adsorption. Phys. Rev. B 71, 224422 (2005).

62. Katayama, K. et al. Orientation control and domain structure analysis of $\{100\}$ oriented epitaxial ferroelectric orthorhombic $\mathrm{HfO}_{2}$-based thin films. J. Appl. Phys. 119, 134101 (2016)

63. Shimizu, T. et al. Ferroelectricity mediated by ferroelastic domain switching in $\mathrm{HfO}_{2}$-based epitaxial thin films. Appl. Phys. Lett. 113, 212901 (2018).

64. Jenkins, M. A. et al. Determination of hafnium zirconium oxide interfacial band alignments using internal photoemission spectroscopy and x-ray photoelectron spectroscopy. ACS Appl. Mater. Interfaces 13, 14634-14643 (2021).

65. Kresse, G. \& Joubert, D. From ultrasoft pseudopotentials to the projector augmented-wave method. Phys. Rev. B 59, 1758 (1999).
66. Perdew, J. P., Burke, K. \& Ernzerhof, M. Generalized gradient approximation made simple. Phys. Rev. Lett. 77, 3865-3868 (1996).

67. Monkhorst, H. J. \& Pack, J. D. Special points for Brillouin-zone integrations. Phys Rev. B 13, 5188 (1976).

\section{ACKNOWLEDGEMENTS}

The authors thank Dr. Andrei Zenkevich for helpful discussions. This work was supported by the National Natural Science Foundation of China (Grant No. 12072307) and the Outstanding Youth Science Foundation of Hunan Province, China (Grant No. 2021JJ20041). The research at University of Nebraska-Lincoln was supported by the National Science Foundation (NSF) through Materials Research Science and Engineering Center (MRSEC) (NSF Grant No. DMR-1420645).

\section{AUTHOR CONTRIBUTIONS}

Q.Y., L.L.T., and E.Y.T. designed the research. Z.J.C. and Q.Y. performed the firstprinciple calculations and wrote the manuscript. All authors discussed the results and reviewed the manuscript. E.Y.T. supervised the project.

\section{COMPETING INTERESTS}

The authors declare no competing interests.

\section{ADDITIONAL INFORMATION}

Supplementary information The online version contains supplementary material available at https://doi.org/10.1038/s41524-021-00679-2.

Correspondence and requests for materials should be addressed to Qiong Yang Lingling Tao or Evgeny Y. Tsymbal.

Reprints and permission information is available at http://www.nature.com/ reprints

Publisher's note Springer Nature remains neutral with regard to jurisdictional claims in published maps and institutional affiliations.

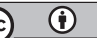

Open Access This article is licensed under a Creative Commons Attribution 4.0 International License, which permits use, sharing, adaptation, distribution and reproduction in any medium or format, as long as you give appropriate credit to the original author(s) and the source, provide a link to the Creative Commons license, and indicate if changes were made. The images or other third party material in this article are included in the article's Creative Commons license, unless indicated otherwise in a credit line to the material. If material is not included in the article's Creative Commons license and your intended use is not permitted by statutory regulation or exceeds the permitted use, you will need to obtain permission directly from the copyright holder. To view a copy of this license, visit http://creativecommons. org/licenses/by/4.0/.

(c) The Author(s) 2021 